\title{
Creencias y estrategias para el control del peso, satisfacción con la imagen corporal y autoestima
}

\author{
Margarita Salvador, Carmen García-Gálvez y Manuel de la Fuente \\ Universidad de Almería (España)
}

Presentamos un estudio descriptivo mediante encuestas con diseño transversal, cuyo objetivo global fue estudiar las creencias y estrategias utilizadas por los estudiantes para el control del peso, la satisfacción/insatisfacción con su imagen corporal y sus relaciones con la autoestima. La muestra estuvo formada por 740 estudiantes de la Universidad de Almería, seleccionada por un procedimiento de muestreo estratificado por sexo, curso y centro, que cumplimentaron el Cuestionario de Creencias y Estrategias para el Control del Peso, elaborado ad hoc para esta investigación, y el Cuestionario de Autoestima de Rosenberg. Los resultados generales muestran que el $73.7 \%$ de la muestra total, no utiliza estrategias para controlar el peso y que sólo el $48.2 \%$ de la muestra total, se esfuerzan por controlarlo. El mayor porcentaje de mujeres se identifica con el prototipo físico actual de pícnicas y el prototipo más deseado es el de atléticas. El porcentaje mayor de los hombres se identifican con en el prototipo actual atlético y es este prototipo el más deseado. El $48.16 \%$ de los sujetos están satisfechos con su prototipo actual y el $51.84 \%$ se muestran insatisfechos. Los hombres están significativamente más satisfechos con su imagen corporal que las mujeres y los sujetos más satisfechos presentan una mayor autoestima.

Palabras clave: Estrategias control del peso, satisfacción/insatisfacción corporal, imagen corporal, autoestima.

Beliefs and strategies for weight management, satisfaction with body image and selfesteem. We present a descriptive research study with crossover design, the overall aim was to study the beliefs and strategies used by students for weight control, satisfaction / dissatisfaction with their body image and relations with self-esteem. The sample consisted of 740 students at the University of Almeria, selected by a stratified sampling procedure by gender, course and facility, which completed the Questionnaire of Beliefs and Strategies for Weight Management, developed ad hoc for this research, and Rosenberg Self-Esteem Questionnaire. The overall results show that $73.7 \%$ of the total sample does not use strategies to control weight, and only $48.2 \%$ of the total sample, they try to control it. The highest percentage of women identified with the actual physical prototype and the prototype fleshy most desired is the Athletic. The largest percentage of men are identified in the current prototype this prototype is athletic and the most desired. The $48.16 \%$ of the subjects are satisfied with their current prototype and the $51.84 \%$ shown is unsatisfied. Men are significantly more satisfied with their body image than women and more satisfied subjects have higher self-esteem.

Key words: Satisfaction/corporal dissatisfaction, corporal Image, self-esteem.

Correspondencia: Margarita Salvador Granados. Universidad de Almería. Departamento de Psicología Evolutiva y de la Educación. Facultad de Psicología. Ctra. de Sacramento s/n. C.P. 04120. La Cañada de San Urbano. Almería (España). E-mail: msalva@ual.es 
Desde los primeros estudios sobre el fenómeno alimentario y sus trastornos llevados a cabo por Cannon y Washburn (1912) y Turró (1912), las investigaciones en este campo han integrado diferentes perspectivas. En la actualidad, encontramos una perspectiva fisiológica y biológica de la conducta alimentaria claramente consolidada, y una perspectiva psicológica, social y educativa de gran relevancia, que plantea que patologías como la obesidad, o la anorexia y la bulimia, propias de una sociedad de la abundancia, requieran que el fenómeno de la alimentación tenga que abordase desde perspectivas de análisis e investigación confluentes y complementarias (López-Espinoza, 2007). Esto ha conducido a incluir, en la comprensión y explicación de las conductas relacionadas con la alimentación y la nutrición, la función determinante que tienen las creencias y los hábitos alimentarios (Behar e Icaza, 1972; Burgess y Dean, 1963; Hurtado, 1990). Además, desde una perspectiva psicológica, el sobrepeso y la obesidad ya desde la infancia, se asocia a conductas alimentarias de riesgo, a alteraciones de la imagen corporal y a creencias erróneas sobre su posible control (Amigo, Fernández, Rodríguez y Rodríguez, 2005; Ballester y Guirado, 2003; Espina, Ortego, Ochoa, Yenes y Alemán, 2001; Sepúlveda, Botella y León, 2001).

Aunque los conocimientos sobre nutrición son indispensables para realizar cambios adecuados en los hábitos alimentarios, no podemos despreciar el papel de la motivación para generar dichos cambios, ya que las nuevas pautas alimentarias y las prescripciones que se establezcan sólo serán eficaces si el individuo ha aceptado la necesidad de cambiar y está motivado para hacerlo. Es por ello, fundamental que se reconozca a los factores psicológicos como ineludibles en cualquier intervención que pretenda basarse en las creencias y los hábitos alimentarios (Behar e Icaza, 1972; Burgess y Dean, 1963; Hurtado, 1990).

Tampoco podemos ignorar que el pertenecer a diversos grupos sociales hace que las conductas y actitudes colectivas ejerzan una gran influencia sobre las creencias y hábitos alimenticios. Se ha observado que el patrón alimentario varía según el grado de escolaridad y el nivel educativo de las personas y las familias, como lo muestran algunos estudios en los que no solo aparecen los patrones alimentarios modificados según la escolaridad, sino que también varían el horario de las comidas, los métodos de preparación y el almacenamiento (Flores, Bresanni y Elias, 1973).

El prestigio social es otro de los factores sociales que determinan las creencias y hábitos alimentarios. Investigaciones con adolescentes (Baile y Garrido, 1999 y Rodríguez y Cruz, 2008) han puesto de manifiesto que son, precisamente, los factores socioculturales y psicológicos los que ejercen una gran influencia sobre la imagen de la mujer y que, incluso, algunos alimentos gozan de significado social (Behar e Icaza, 1972; Burgess y Dean, 1963).

Para Baile y Garrido (1999), lo importante no es solo comprobar que las adolescentes utilizan formas de pensamiento erróneas en relación con su imagen, sino 
que de esos procesamientos se derivan pensamientos concretos que pasan a ser valores en su vida, que producen una actitud de cambio para intentar ser como el modelo de belleza delgado, que impera en el ideal social. Según Moreno, Cervelló y Moreno, (2008), en la sociedad occidental se cultiva mucho el cuerpo y la imagen corporal considerándolos, como un instrumento muy importante para presentarnos a los demás.

En este sentido, Ballester y Guirado (2003) observaron que el tener que ajustarse a ciertos valores e ideales de belleza homogeneizados en el entorno social, lleva a niños y niñas, y en particular a estas últimas, a un estado de evaluación corporal continúo, en edades cada vez más tempranas (11 años), lo que les lleva a desarrollar comportamientos que atentan contra su salud física y psicológica. Es comúnmente aceptado que la imagen física tiene una gran importancia en nuestra sociedad y en nuestra época, pero la consecución del "cuerpo perfecto" conlleva conductas de riesgos (utilización de dietas milagro, dudosos alimentos sin calorías, cremas adelgazantes, excesivo ejercicio sin control, etc.), que ponen en serio peligro la salud.

Como plantean Merino, Pombo y Godás (2001), la cultura de la delgadez en la que actualmente nos vemos inmersos establece un estereotipo corporal excesivamente delgado, capaz de desencadenar una serie de valores y normas que determinan una cadena de comportamientos relacionados con la talla, peso y figura corporal, comportamientos que, muy probablemente, conducirán al desarrollo de un problema o trastorno alimentario.

Muchas personas creen que sus problemas sociales o personales se solucionarían con un mejor aspecto físico y esto es especialmente relevante en las mujeres, que reciben más presiones sociales y culturales, haciéndolas más susceptibles y provocando en ellas una mayor insatisfacción con su imagen corporal. Este proceso es especialmente relevante, si se tiene en cuenta que la variable satisfacción/insatisfacción con la imagen corporal está considerada como uno de los factores de riesgo asociados con los trastornos de la alimentación (Fabian y Thompson, 1989; Leon, Fulkerson, Perry y Cudeck, 1993).

En este sentido, diversos estudios indican que las chicas presentan una mayor insatisfacción con la imagen corporal, evalúan negativamente su apariencia y muestran mayor miedo a "estar gorda" o ganar peso (Cash y Henry, 1995; Lameiras, Calado, Rodríguez y Fernández, 2003). De igual forma, las mujeres tienen mayor preocupación por su cuerpo y su imagen, son más críticas con sus cuerpos y están más involucradas que los hombres en la apariencia física (Bane y McAuley, 1998; Gómez-Peresmitré y Acosta, 2002; Heunemamm, Shapiro, Hampton y Mitchell, 1966; Loland, 1998). Sin embargo, no existe un consenso en lo que se refiere a la relación entre insatisfacción corporal y cultura, ya que adolescentes de otras culturas, como las latinoamericanas, se muestran significativamente más influenciadas por el modelo estético corporal que las españolas (Rodríguez y Cruz, 2008). 
La imagen corporal incluye lo que cada uno piensa, siente, y cómo se percibe y actúa, en relación con su propio cuerpo (Cash, 1994; Cash y Pruzinsky, 1990). Shilder (1935), planteaba que una parte significativa de la imagen corporal permanece fuera de la consciencia aunque influyendo en la conducta de la persona y adelantándose a líneas actuales de investigación, defendía que la imagen corporal es un constructo que refleja deseos, actitudes, emociones, cogniciones e interacciones con otras personas. De igual modo, Pruzinsky y Cash (1990), defendían que la imagen corporal es una experiencia totalmente subjetiva y personalizada que puede no ser congruente con la realidad objetiva, cuyo componente emocional incluye experiencias de satisfacción/insatisfacción asociada con nuestra apariencia o experiencia corporal.

Por lo tanto, la imagen corporal se considera una parte del autoconcepto (Mock, 1993), entendido como el conjunto de percepciones o referencias que la persona tiene de sí misma, que incluye juicios acerca de comportamientos, de habilidades o de la apariencia externa (Shavelson y Bolus, 1992). Las valoraciones, positivas ó negativas, realizadas por la persona sobre ese conjunto de percepciones, y los sentimientos que le producen, determinan su autoestima. Por tanto, la imagen corporal está íntimamente relacionada con la autoestima, en el sentido considerado por Rosenberg (1965) de una actitud/sentimiento positivo o negativo hacía uno mismo, basada en la evaluación de las propias características, que incluye sentimientos de satisfacción/insatisfacción, hasta el punto de que según Fox (2000) el grado de aceptación personal de la imagen corporal determina el desarrollo de la autoestima. Esto implica que una pobre imagen corporal se asocie con una baja autoestima (Calado, Lameiras y Rodríguez, 2004).

Quizás uno de los momentos evolutivos más significativos en esta relación sea la adolescencia, donde podemos comprobar que los jóvenes experimentan malestar psicológico debido a su físico durante toda la etapa, al margen de si el concepto que tienen de su físico es bajo, medio o alto, y que los chicos se sienten en general mejor que las chicas puesto que el deporte parece ser una buena forma de minimizar ese malestar psicológico, probablemente porque mejora el autoconcepto físico (Rodríguez-Fernández, 2008).

Consecuentemente, si se entiende la imagen corporal como la evaluación tanto cognitiva como emocional que se tiene del propio cuerpo en un momento determinado, y se considera la autoestima como las evaluaciones, positivas y negativas, que la persona tiene de sí misma, se puede concluir que aceptarnos y valorarnos positivamente es importante, porque el hecho de sentirnos bien con nosotros mismos, puede influir positivamente sobre nuestra forma de actuar y de controlar nuestro comportamiento.

Desde este planteamiento investigador, diseñamos el presente estudio, en el marco de un proyecto de investigación más amplio realizado por el Grupo PESSO de la Universidad de Almería. Nuestro objetivo fue describir conductas, hábitos y estrategias 
relacionadas con la alimentación en universitarios andaluces. Los objetivos que se plantearon para estudio fueron:

Objetivo 1. Conocer las creencias que tienen los sujetos sobre la alimentación y el control del peso y sobre las estrategias para conseguirlo.

Objetivo 2. Analizar los patrones físicos de identificación actual y deseada, su grado de coincidencia a través del Índice de Satisfacción (IS) y sus relaciones con el Índice de Masa Corporal (IMC), edad y sexo.

Objetivo 3. Estudiar las relaciones de la Autoestima con el sexo, la edad, el Índice de Satisfacción (IS) y el Índice de Masa Corporal (IMC).

\section{METODO}

\section{Participantes}

En el estudio participaron 740 estudiantes de la Universidad de Almería, 505 mujeres (68.24\%) y 235 hombres (31.75\%), con una edad media de 21.15 años, pertenecientes a las facultades de Humanidades $(N=350,46.6 \%)$, Económicas y Empresariales $(N=209,27.7 \%)$, Experimentales $(N=43,5.7 \%)$ y a la Escuela Politécnica Superior $(N=148,20 \%)$. La muestra fue seleccionada por un procedimiento de muestreo estratificado por sexo, curso y centro. La muestra obtenida se consideró adecuada por cumplir los criterios de representación muestral (Mellor, Rapoport y Maliniak, 2008). La distribución de los estudiantes por centros y sexo, se presenta en la tabla 1.

\begin{tabular}{cccccc}
\multicolumn{6}{c}{ Tabla 1. Distribución de la muestra por centros y sexo } \\
\hline \multicolumn{7}{c}{ Hum. } & EE. & Exper. & EPS & $\%$ \\
\hline Hombres & 84 & 48 & 20 & 61 & $31.9 \%$ \\
Mujeres & 266 & 161 & 23 & 87 & $68.1 \%$ \\
$\%$ & $46.6 \%$ & $27.7 \%$ & $5.7 \%$ & $20 \%$ & $100 \%$ \\
\hline
\end{tabular}

La distribución de los sujetos en función de su edad, según las tres categorías establecidas para este estudio, fue la siguiente: muy jóvenes, que agrupa a los sujetos con edades entre 18 y 20 años $(N=453,61.21 \%)$; jóvenes, con edades entre 21 y 30 años $(N=258,34.86 \%)$ y adultos, con edades entre 31 y 50 años $(N=29,3.91 \%)$.

\section{Instrumentos}

Cuestionario de Creencias y Estrategias para el Control del Peso (Salvador et al., 2008), que fue elaborado ad hoc para esta investigación. Es un cuestionario de autoinforme, estructurado en los siguientes partes:

a.-. Datos personales, incluía los siguientes ítems: edad, peso, altura, titulación, sexo, zona en la que vive y nivel de estudios del padre y la madre. A partir de los datos de peso y altura se calculó el Índice de masa corporal (IMC), dividiendo el peso por el cuadrado de la talla. 
b.- Cuestiones generales, formada por 9 ítems que evaluaban creencias ( 2 ítems) y estrategias (3 ítems) relacionados con la alimentación, el control del peso y otros factores relacionados (4 ítems).

c.- Identificación del prototipo físico actual y deseado (con el que les gustaría identificarse). Se utilizaron tres dibujos, de hombre y mujer, de los prototipos clásicos de leptocúrtico, pícnico y atlético, basados en los trabajos de Acosta y Gómez-Peresmitré (2003) y de Williamson et al. (2000), autores que utilizan un procedimiento similar con mayor número de modelos gráficos. A partir de la identificación realizada sobre el prototipo físico actual y sobre el deseado, se calculó el Índice de Satisfacción (IS), con dos categorías: 1 . Satisfechos, cuando coincidía el prototipo con el que se identificaban en la actualidad con el prototipo con el que le gustaría identificarse y 2. Insatisfechos, cuando no de daba esta coincidencia.

d.- Evaluación de las creencias de utilidad y de utilización de estrategias de control del peso, formada por un listado de 34 estrategias. Los sujetos debían indicar si creían que servían/no servían para controlar el peso y decir si las utilizaban o no, en la actualidad o en el pasado, para conseguir el control de su peso.

e.- Escala de Autoestima de Rosenberg (1965) (Adaptación española de Martín-Albo, Núñez, Navarro y Grijalva, 2007). Evalúa la autoestima global y tiene un coeficiente alpha de Cronbach de 0.85 (Martín-Albo et al., 2007). Consta de 10 ítems contestados en una escala tipo Likert cuya puntuación va desde 1 (muy de acuerdo) hasta 4 (muy en desacuerdo). A mayor puntuación, mayor es el grado de autoestima ó grado de satisfacción que una persona tiene consigo misma. La mitad de los ítems están redactados positivamente y la otra mitad negativamente. Para su corrección deben invertirse las puntuaciones de los ítems enunciados negativamente y sumarse la puntuación de todos los ítems, pudiéndose obtener una puntuación directa que oscila entre 10 y 40 puntos. En este estudio se ha utilizado un Índice de Autoestima (IA), calculado a partir de las puntuaciones medias.

\section{Procedimiento}

La presente investigación, de carácter exploratorio y representativo, es un estudio descriptivo transversal mediante encuestas, comúnmente utilizado en estudios evolutivos (Salvador, De la Fuente y Alvarez, 2009; Gázquez et al., 2009).

El cuestionario se administró colectivamente en clase, en horario lectivo y a los grupos naturales, durante el primer trimestre del curso académico 2008-2009. Todos los sujetos participaron de forma voluntaria. Los encuestadores preparados para esta investigación, dieron una información previa sobre este estudio y las instrucciones específicas para la cumplimentación de los cuestionarios. El tiempo aproximado de ejecución fue de 20 minutos. 


\section{RESULTADOS}

Se han utilizado estadísticos descriptivos, como medias y porcentajes, y se han realizado correlaciones y análisis de comparación de medias. Además se han calculado los Índices de Masa Corporal (IMC), el Índice de Satisfacción (IS) y el Índice de Autoestima (IA). Para el análisis de los datos se utilizó el paquete estadístico SPSS versión 15.0.

Para una presentación ordenada de los resultados y para una mejor comprensión, se agrupan en función de los objetivos del estudio.

Tabla 2. \% de respuestas afirmativas

\begin{tabular}{ll}
\hline ¿Crees que es importante alimentarse bien? & $99.3 \%$ \\
¿Crees que es necesario controlar el peso? & $92.6 \%$ \\
Habitualmente vives con tus padres & $63.5 \%$ \\
Realizas alguna actividad física & $49.9 \%$ \\
¿Haces esfuerzos por controlar tu peso? & $46.9 \%$ \\
Realizas tú mismo/a la elabora de tus comidas & $45.7 \%$ \\
¿Utilizas estrategias (trucos) para controlar tu peso? & $26.3 \%$ \\
Eres fumador/a & $21.8 \%$ \\
¿En los últimos 6 meses ha estado sometido a dieta? & $14.1 \%$ \\
\hline
\end{tabular}

Objetivo 1: Conocer las creencias que tiene los sujetos y las estrategias que utilizan para conseguir el control de su peso

De las cuestiones generales preguntadas, obtuvimos una primera información sobre creencias y estrategias relacionadas con su alimentación y control del peso. En la tabla 2 se presentan los porcentajes de respuestas afirmativas a cada una de las cuestiones.

Los datos nos revelan que aunque creen que es importante alimentarse bien (99.3\%) y que es necesario controlar el peso (92.6\%), sin embargo el $73.7 \%$ de la muestra evidencia que no utiliza estrategias para controlar su peso, no se esfuerza en controlar su peso el $52.8 \%$ y no ha llevado a cabo ninguna dieta en los últimos seis meses el $85.8 \%$. Como factores relevantes por su incidencia en el peso, es de interés que un $49.9 \%$ realice alguna actividad física y que un $78.2 \%$ no fume. Por último, por su relación con los hábitos y estilos de alimentación, es relevante que el $63.5 \%$ de los sujetos vivan con sus padres, por lo que significa de seguimiento de estilos y pautas de alimentación del grupo familiar, y que sólo el $45.7 \%$ se elabora sus comidas, con un control directo de su alimentación.

La ordenación de los porcentajes de respuesta dadas a cada estrategia, según la creencia de utilidad o inutilidad para controlar el peso y según su utilización o no por los sujetos, permitió identificar las diez estrategias más valoradas y utilizadas y las diez estrategias menos valoradas y menos utilizadas, que se presentan en las tablas 3 y 4.

El análisis de las estrategias que los sujetos creen que más sirven y que más utilizan para el control de su peso, pone de manifiesto que hay una alta relación entre 
ambos conjuntos de valoraciones, ya que nueve estrategias aparecen comunes. La estrategia que creen los sujetos que más sirve es la de "ejercicios aeróbicos" (un 94.2\%), pero es utilizada por un $68.8 \%$, o en el caso de "comer con moderación", creen que sirve un $92.7 \%$ y la utilizan el $76.7 \%$. Un $89.1 \%$ de los sujetos creen que "No comer alimentos basura" sirve para controlar el peso, pero sólo la utilizan un 65.3\%, o "No tomar alcohol" creen que sirve un $76.2 \%$ y la utilizan un $45.6 \%$. Con estas comparaciones puede constatarse las diferencias entre los porcentajes de las creencias y los de las utilizaciones de las estrategias.

Tabla 3. Estrategias que creen que sirven y estrategias que utilizan

\begin{tabular}{llll}
\hline \multicolumn{1}{c}{ sirven } & \multicolumn{2}{c}{ utilizan } \\
\hline Ejercicios aeróbicos & $94.2 \%$ & Comer con moderación & $76.7 \%$ \\
Comer con moderación & $92.7 \%$ & Beber mucha agua & $72.9 \%$ \\
No comer alimentos basura & $89.1 \%$ & Ejercicios aeróbicos & $68.6 \%$ \\
Beber mucha agua & $87.6 \%$ & No comer alimentos basura & $65.3 \%$ \\
No comer bollería o chocolate & $76.6 \%$ & No comer bollería o chocolate & $57.3 \%$ \\
No tomar alcohol & $76.2 \%$ & Alimentos ricos en fibras & $49.7 \%$ \\
Alimentos ricos en fibras & $75.3 \%$ & No tomar alcohol & $45.6 \%$ \\
Gimnasio & $44.7 \%$ & No tomar grasa & $31.6 \%$ \\
Dieta de proteínas & $40.7 \%$ & Comer pescado en vez de carne & $28.9 \%$ \\
Comer pescado en vez de carne & $40.1 \%$ & Gimnasio & $28.4 \%$ \\
\hline
\end{tabular}

Tabla 4. Estrategias que creen que no sirven y estrategias que no utilizan

\begin{tabular}{lclc}
\hline \multicolumn{1}{c}{ no sirven } & \multicolumn{2}{c}{ no utilizan } \\
\hline No desayunar & $98.5 \%$ & Liposucción & $99.2 \%$ \\
Comer solo tapas & $98 \%$ & Acupuntura & $98.1 \%$ \\
Vomitar comidas & $96.9 \%$ & Vomitar comidas & $97.3 \%$ \\
Comer una vez al día & $96.4 \%$ & Comer solo tapas & $97.1 \%$ \\
Parches reductores & $95.1 \%$ & Parches reductores & $96.7 \%$ \\
No comer carnes & $93.8 \%$ & Laxantes & $96 \%$ \\
Laxantes & $93.8 \%$ & Pastillas adelgazantes & $95.6 \%$ \\
No comer comidas cuchara & $93.5 \%$ & Comer una vez al día & $94.6 \%$ \\
Dieta de un solo alimento & $93 \%$ & Dieta de un solo alimento & $93.8 \%$ \\
Pastillas adelgazantes & $92.7 \%$ & No comer carnes & $92.7 \%$ \\
\hline
\end{tabular}

La identificación de las diez estrategias que los sujetos creen que no sirven y que dicen no utilizar para el control de su peso, también pone de manifiesto la alta relación entre ambos conjuntos de valoraciones, pero destaca la alta homogeneidad de los porcentajes entre ambas, lo que pone de manifiesto, a su vez, la alta correspondencia que hay entre las creencias que tienen los sujetos sobre las estrategias que no sirven y las que no ponen en práctica, para controlar su peso.

Objetivo 2. Analizar los patrones físicos de identificación actual y deseada, su grado de coincidencia a través del Índice de Satisfacción y sus relaciones con el Índice de Masa CorporaI (IMC), edad y sexo

El análisis de las identificaciones que realizaron los sujetos de sus prototipos físicos actuales (Parte 3 del Cuestionario de Creencias y Estrategias para el Control del Peso), reveló que el prototipo actual con el que más sujetos se identifican es el pícnico con un $39.2 \%$ de los sujetos de la muestra, seguido del leptocúrtico, con un $30.9 \%$ y del 
atlético, con un $29.9 \%$. Los prototipos físicos deseados reflejan que a un $66.0 \%$ de los sujetos les gustaría ser atléticos, a un $26.6 \%$, leptocúrticos y a un $7.4 \%$, pícnicos. Los datos de los prototipos actuales y deseados se presentan en la tabla 5.

Tabla 5. Identificación de los prototipos físicos actuales y deseados por sexos

\begin{tabular}{lcccccc}
\hline & \multicolumn{3}{c}{ Prototipo físico actual } & \multicolumn{3}{c}{ Prototipo físico deseado } \\
\cline { 2 - 7 } & $\%$ muestra & $\%$ mujeres & \% hombres & \% muestra & $\%$ mujeres & $\%$ hombres \\
\hline Leptocúrtico & $30.9 \%$ & $30.8 \%$ & $30.9 \%$ & $26.6 \%$ & $33.4 \%$ & $11.7 \%$ \\
Pícnico & $39.2 \%$ & $42.4 \%$ & $32.6 \%$ & $7.4 \%$ & $6.4 \%$ & $9.6 \%$ \\
Atlético & $29.9 \%$ & $26.8 \%$ & $36.5 \%$ & $66.0 \%$ & $60.2 \%$ & $78.7 \%$ \\
\hline
\end{tabular}

Un análisis por sexos reflejó que el $42.4 \%$ de las mujeres se identifican con el prototipo actual pícnico, el $30.8 \%$ con el leptocúrtico y un $26.8 \%$ con el atlético, mientras que el $36.5 \%$ de los hombres se identifican con el prototipo atlético, el $32.6 \%$ con el pícnico y el $30.8 \%$ con el leptocúrtico. Los prototipos deseados fueron: a un $60.2 \%$ de las mujeres les gustaría ser atléticas, al 33.4\%, leptocúrticas y al $6.4 \%$, pícnicas y que a un $78.7 \%$ de los hombres les gustaría ser atléticos, a un $11.7 \%$, leptocúrticos y a un $9.6 \%$, pícnicos.

Los prototipos deseados a partir de los prototipos actuales, para hombres y mujeres, se presenta en la tabla 6. Estos datos ponen de evidencia que las mujeres leptocúrticas, en su mayoría quieren seguir siendo este prototipo físico (17.3\%), las pícnicas desean ser mayoritariamente atléticas (23.3\%), y las atléticas eligen ese mismo prototipo, en un mayor porcentaje (23.4\%). Por su parte, los hombres leptocúrticos eligen este prototipo, mayoritariamente $(20.2 \%)$, los pícnicos desean ser atléticos en su mayoría (23.1\%), y la casi totalidad de los atléticos eligen seguir siéndolo (35.4\%).

Tabla 6. Relación entre prototipo físico actual y deseado por sexos

\begin{tabular}{llcccc}
\hline & \multicolumn{3}{c}{ Prototipo actual } & \multicolumn{3}{c}{ Prototipo deseado } & \\
\cline { 2 - 6 } & & & Leptocúrtico & Pícnico & Atlético \\
\hline \multirow{4}{*}{ Mujer } & Leptocúrtico & $30.8 \%$ & $17.3 \%$ & $0 \%$ & $13.5 \%$ \\
& Pícnico & $42.4 \%$ & $14.3 \%$ & $4.8 \%$ & $23.3 \%$ \\
& Atlético & $26.8 \%$ & $2 \%$ & $1.4 \%$ & $23.4 \%$ \\
& Total & $100 \%$ & $33.6 \%$ & $6.2 \%$ & $60.2 \%$ \\
\hline \multirow{5}{*}{ Hombre } & Leptocúrtico & $30.9 \%$ & $10.2 \%$ & $0.4 \%$ & $20.2 \%$ \\
& Pícnico & $32.6 \%$ & $0.4 \%$ & $9.1 \%$ & $23.1 \%$ \\
& Atlético & $36.5 \%$ & $1.1 \%$ & $0 \%$ & $35.4 \%$ \\
& Total & $100 \%$ & $11.7 \%$ & $9.6 \%$ & $78.7 \%$ \\
\hline
\end{tabular}

El análisis de las identificaciones realizadas por los sujetos sobre los prototipos físicos actuales y deseados, en función de los distintos grupos de edad (Tablas 7 y 8), indica que el mayor porcentaje de los sujetos que identifican con el prototipo leptocúrtico son los muy jóvenes $(33.1 \%)$. Entre los sujetos que se categorizan como pícnicos, el mayor porcentaje lo representan los adultos $(51.75 \%)$ y el grupo que más se identifican con el prototipo atlético es el de los jóvenes (32.95\%). A su vez, los sujetos que en mayor porcentaje desearían ser leptocúrticos son los muy jóvenes (29.7\%), los 
que más desearían ser pícnicos son los adultos (8.2\%) y los que más se identifican con el prototipo deseado atlético son los adultos (79.3\%).

Tabla 7. Identificación prototipo físico actual por grupos de edad

\begin{tabular}{lcccc}
\hline & $\%$ muestra & \% adultos & \% jóvenes & $\%$ muy jóvenes \\
\hline Leptocúrtico & $30.9 \%$ & $24.10 \%$ & $27.95 \%$ & $33.10 \%$ \\
Pícnico & $39.2 \%$ & $51.75 \%$ & $39.10 \%$ & $38.60 \%$ \\
Atlético & $29.9 \%$ & $24.15 \%$ & $32.95 \%$ & $28.30 \%$ \\
\hline
\end{tabular}

Tabla 8. Identificación prototipo físico deseado por grupos de edad

\begin{tabular}{lcccc}
\hline & $\%$ muestra & $\%$ adultos & \% jóvenes & $\%$ muy jóvenes \\
\hline Leptocúrtico & $26.6 \%$ & $13.8 \%$ & $22.7 \%$ & $29.7 \%$ \\
Pícnico & $7.4 \%$ & $6.9 \%$ & $8.2 \%$ & $6.7 \%$ \\
Atlético & $66 \%$ & $79.3 \%$ & $69.1 \%$ & $63.6 \%$ \\
\hline
\end{tabular}

A partir de la identificación de las coincidencias/no coincidencias en las elecciones del prototipo físico actual y del deseado de cada sujeto, se ha obtenido un índice global de satisfacción (IS), cuyo valor es 0.51 (rango entre 0 y 1). El IS del grupo de mujeres es de 0.46 y el de hombres es de 0.54. Para comprobar si había diferencias significativas entre mujeres y hombres se calculó la t de Student para muestras independientes, obteniendo una $t=2.109(p<.05)$, lo que refleja que los hombres están significativamente más satisfechos que las mujeres.

De la muestra global, encontramos que el $48.16 \%$ está satisfecha y coincide en la elección de su prototipo actual y deseado, mientras que el $51.84 \%$ está insatisfecha.

Como se puede ver en la tabla 9 , de entre los sujetos satisfechos encontramos el mayor porcentaje en los atléticos $(91.2 \%)$ y entre los sujetos insatisfechos son los pícnicos los que presentan un mayor porcentaje (84.48\%).

Tabla 9. Índice de Satisfacción: Prototipo físico actual versus prototipo físico deseado

\begin{tabular}{lcccc}
\hline & \multirow{2}{*}{$\%$ muestra } & \multicolumn{3}{c}{ Prototipo actual } \\
\cline { 3 - 5 } & & Leptocúrtico & Pícnico & Atlético \\
\hline Satisfecho & $48.16 \%$ & $48.91 \%$ & $15.52 \%$ & $91.20 \%$ \\
Insatisfecho & $51.84 \%$ & $51.09 \%$ & $84.48 \%$ & $8.80 \%$ \\
\hline
\end{tabular}

Tabla 10. Índice de Satisfacción: hombres y mujeres

\begin{tabular}{lcccc}
\hline & \multicolumn{4}{c}{ mujeres } \\
\hline \multirow{2}{*}{$\%$ mujeres } & \multicolumn{3}{c}{ Prototipo actual } \\
\cline { 3 - 5 } & & Leptocúrtico & Pícnico & Atlético \\
\hline Satisfecho & $45.95 \%$ & $56.13 \%$ & $11.37 \%$ & $87.41 \%$ \\
Insatisfecho & $54.05 \%$ & $43.87 \%$ & $88.63 \%$ & $12.59 \%$ \\
\hline & & hombres & Prototipo actual & \\
\hline \multirow{2}{*}{$\%$ hombres } & & Pícnico & Atlético \\
\cline { 3 - 5 } & $53.41 \%$ & Leptocúrtico & $27.27 \%$ & $97.53 \%$ \\
Insatisfecho & $46.59 \%$ & $33.33 \%$ & $72.73 \%$ & $2.47 \%$ \\
\hline
\end{tabular}


El análisis de los IS, por sexos puso de manifiesto que el $53.41 \%$ del total de los hombres frente al $45.95 \%$ del total de las mujeres, pertenecen a la categoría de satisfechos, mientras que el $54.05 \%$ de las mujeres frente al $46.59 \%$ de los hombres se incluyen en la categoría de insatisfechas. Considerando el total de la muestra, encontramos que las mujeres satisfechas con su prototipo actual representan un $31.3 \%$ del total de sujetos y las insatisfechas, un 37.2\%, y los hombres satisfechos con su prototipo actual representan un $17.0 \%$ de la muestra y los no satisfechos, un $14.5 \%$.

El desglose más detallado de los IS de hombres y mujeres se presenta en la tabla 10. Tanto en los hombres como en las mujeres, en la categoría de satisfechos, el prototipo que mayor porcentaje representa es atlético ( $\mathrm{V}=87.41 \%, \mathrm{H}=97.53 \%)$, e igualmente, en ambos sexos, en la categoría de insatisfechos, la mayor representación la tiene el prototipo pícnico ( $\mathrm{V}=88.63 \%, \mathrm{H}=72.73 \%)$.

Para poder relacionar el IS con el IMC, de la Parte 2 del Cuestionario de Creencias y Estrategias para el Control del Peso) se obtuvieron los siguientes resultados. El peso medio de la muestra que es de $64.52 \mathrm{Kg}$. Siguiendo el criterio habitualmente utilizado en este tipo de estudios por el que un IMC $>25$ representa un sobrepeso y un IMC $<25$ significa la no existencia de sobrepeso, se identificaron 146 sujetos $(19.4 \%)$ que presentan sobrepeso y $604(80.6 \%)$ que no lo presentan. El IMC medio es de 22.46, el de las mujeres de 21.77 y el de los hombres de 23.93. La distribución en función de las dos categorías del IMC y del sexo es la siguiente: con un IMC $>25$, el $8.84 \%$ son mujeres y el $10.56 \%$ hombres y con un IMC $<25$, el $59 \%$ son mujeres y el $21.52 \%$ hombres.

Para constatar la existencia de relaciones entre el IMC y la variable edad se calculó el coeficiente de correlación de Pearson $(r=.137(.000)$, significativa al nivel .05 (bilateral)). Igualmente, se hizo entre el IMC y la variable sexo $(r=.316(.000)$, significativa al nivel .01 (bilateral)). En ambos casos la relación es positiva, aunque baja.

De manera complementaria a los análisis anteriores, se calcularon los siguientes coeficientes de correlación de Pearson: relación IS con IMC: $r=.248(.000)$, significativa al nivel .01 (bilateral), lo que refleja que los sujetos que tienen una mayor satisfacción con su imagen corporal tienen un IMC $<25$; relación IS con edad: $r=-.047$ (.205), no significativa, que refleja que no hay relación entre la edad y la satisfación/insatisfacción con la imagen corporal; relación IS con sexo: $r=-.078 *$ (.035), significativa al nivel .01 (bilateral), que refleja que una mayor satisfacción se relaciona con los hombres y que una mayor insatisfacción se relaciona con las mujeres.

Objetivo 3. Estudiar las relaciones de la Autoestima con el sexo, la edad, el Índice de Satisfacción (IS) y el Índice de Masa Corporal (IMC)

De la Escala de Autoestima de Rosenberg, se ha obtenido la puntuación global de la muestra en autoestima, que es de 32.04 puntos, que sobre un total de 40 puntos de máxima, representa una autoestima alta. La puntuación media de las mujeres es de 32.10 puntos y la de los hombres, de 33.20. Para homogeneizar los datos de la autoestima con 
los de los índices anteriormente calculados, se han transformado las puntuaciones directas en puntuaciones medias y se ha obtenido el Índice de Autoestima global (IA). Los valores han sido: $(\mathrm{IA})=3.25$; $(\mathrm{IA})$-mujeres= 3.22 e $(\mathrm{IA})$-hombres $=3.32$. Para comprobar si había diferencias significativas entre mujeres y hombres en el (IA) se calculó la t de Student para muestras independientes, obteniendo una $t=-2.489(p<.05)$, lo que refleja que los hombres tienen una mayor autoestima significativamente mayor que las mujeres.

Se han calculado las correlaciones (coeficiente de correlación de Pearson) entre el IA y las siguientes variables: sexo, edad, IS e IMC. La correlación entre IA y sexo tiene una $r=.090 *$ (.013), positiva, muy alta y significativa al nivel .05 (bilateral), que refleja que la mayor autoestima está relacionada con el sexo= hombre. La correlación entre IA con edad tiene una $r=.026$ (.468), no significativa. La correlación entre IA con IS tiene una $r=.170 * *(.000)$, positiva, baja y significativa al nivel .01 (bilateral), lo que significa que los sujetos más satisfechos presentan una mayor autoestima. La correlación entre (IA) con (IMC) tiene una $r=.010$ (.794), no significativa.

\section{DISCUSION}

Con los resultados obtenidos hemos cubierto los tres objetivos planteados para esta investigación. En relación a nuestro primer objetivo, hemos profundizado en el conocimiento de las creencias que tienen los sujetos y de las estrategias que utilizan relacionadas con sus conductas alimentarias y con el control de su peso.

De los datos obtenidos hay que resaltar la falta de correspondencia entre las creencias relacionadas con la alimentación y el control del peso y la realización de acciones y estrategias para conseguirlo. Es de resaltar que un $73.7 \%$ de la muestra no utiliza estrategias para controlar su peso, un $52.8 \%$ no se esfuerza en controlar su peso, un $51.1 \%$ no realiza alguna actividad física y un $85.8 \%$ no ha llevado a cabo ninguna dieta en los últimos seis meses, aunque el $99.3 \%$ cree que es importante alimentarse bien y el $92.6 \%$ cree que es necesario controlar el peso.

La identificación de las diez estrategias que los sujetos creen que más sirven para el control de su peso y de las diez más utilizadas, ha puesto de manifiesto la alta relación que hay entre ambos conjuntos de estrategias. Las cinco estrategias que los sujetos creen que más sirven: ejercicios aeróbicos, comer con moderación, no comer alimentos basura, beber mucho agua y no comer bollería o chocolate (en un rango entre un 94.2 y un $76.6 \%$ ) y las cinco estrategias que los sujetos más utilizan son: comer con moderación, beber mucho agua, ejercicios aeróbicos, no comer alimentos basura y no comer bollería o chocolate (con un rango entre $76.6 \%$ y 53.3\%). Observando los 
porcentajes se puede constatar que los relacionados con la ejecución de estrategias son inferiores a los de las creencias.

Las diez estrategias que los sujetos creen que no sirven y que dicen no utilizar para el control de su peso también presenta una alta correspondencia. Las cinco estrategias que los sujetos creen que sirven menos para controlar su peso son: no desayunar, comer solo tapas, vomitar comidas, comer una vez al día y parches reductores (con un rango de entre $98.5 \%$ y $95.1 \%$ ) y las cinco menos utilizadas son: liposucción, acupuntura, vomitar comidas, comer solo tapas y parches reductores (en un rango entre un $99.2 \%$ y un $96.7 \%$ ).

Relacionado con el segundo objetivo del estudio, se ha analizado y descrito el conjunto de patrones físicos de identificación actual y deseada, así como su satisfacción/insatisfacción y sus relaciones con otros índices y variables.

Se ha constatado que el prototipo actual con el que más sujetos se identifican es el pícnico, seguido del leptocúrtico y del atlético, mientras que el prototipo deseado por más sujetos es el atlético, seguido del leptocúrtico y del pícnico. El mayor porcentaje de mujeres se identifica con el prototipo actual de pícnicas y el prototipo más deseado es el de atléticas y el porcentaje mayor de los hombres se presentan en el prototipo actual atlético y se identifican también con el prototipo atlético, como el más deseado.

Del desglose de las elecciones de los prototipos deseados a partir de los prototipos actuales, y considerando los porcentajes más altos, cabe resaltar que las mujeres leptocúrticas quieren seguir siendo este prototipo físico, las pícnicas desean ser atléticas y las atléticas eligen ese mismo prototipo y que, por su parte, los hombres leptocúrticos eligen este prototipo, los pícnicos desean ser atléticos y los atléticos eligen seguir siendo este prototipo.

En función de la edad, y centrándonos en los mayores porcentajes, los sujetos que identifican con el prototipo actual leptocúrtico son los muy jóvenes, con el prototipo pícnico los adultos y con el prototipo atlético los jóvenes. Igualmente, se identifican como prototipo deseado en mayor porcentaje, los jóvenes con el leptocúrtico y los adultos con el pícnico y con el atlético.

A partir de la identificación de las coincidencias/no coincidencias en las elecciones del prototipo físico actual y del deseado de cada sujeto, se ha obtenido un índice global de satisfacción (IS), que para las mujeres es de 0.46 y para los hombres es de 0.54 . Estas diferencias son estadísticamente significativas y reflejan que los hombres están significativamente más satisfechos que las mujeres. Globalmente, el $48.16 \%$ de los sujetos se sienten satisfechos y el $51.84 \%$ se siente insatisfecho. El prototipo actual atlético produce el mayor porcentaje de satisfechos, mientras que el porcentaje mayor de insatisfechos se genera entre los pícnicos.

En función del sexo, el $53.41 \%$ del total de los hombres están satisfechos y el $46.59 \%$ insatisfechos, por el contrario, el $45.95 \%$ del total de las mujeres se muestras 
satisfechas y el $54.05 \%$ insatisfechas. El prototipo que mayor satisfacción produce, tanto en hombres como en mujeres, es el atlético, mientras que el prototipo pícnico es el que más insatisfacción genera en hombres y en mujeres.

El análisis de la correlación entre el IS y la edad reflejó que no hay relación entre la edad y la satisfacción/insatisfacción con la imagen corporal. La correlación entre el IS y el sexo evidenció que una mayor satisfacción se relaciona con los hombres y que una mayor insatisfacción se relaciona con las mujeres.

El cálculo del IMC permitió identificar a un $8.84 \%$ de mujeres y a un $10.56 \%$ de hombres con sobrepeso y a un $59 \%$ de mujeres y a un $21.52 \%$ de hombres sin sobrepeso. Se encontró una correlación significativa entre el IMC y el IS reflejando que los sujetos que tienen una mayor satisfacción con su imagen corporal son los que no tienen sobrepeso. Igualmente, el IMC correlaciona significativamente con la edad, reflejando que a mayor edad se produce un mayor IMC. Además, las relaciones entre el IMC y el sexo, evidenciando que el sobrepeso está relacionado con los hombres y la ausencia de sobrepeso, con las mujeres.

En relación con nuestro tercer objetivo, se han analizado las relaciones de la autoestima con los índices anteriormente obtenidos y con otras variables relevantes. Se ha encontrado que los hombres tienen, significativamente, mayor autoestima que las mujeres, que la mayor autoestima está relacionada con el sexo $=$ hombre y que los sujetos más satisfechos presentan, significativamente, una mayor autoestima. Por el contrario, la autoestima no presentó correlaciones significativas ni con el IMC, ni con la edad.

Globalmente, los datos obtenidos son coincidentes con investigaciones anteriores. Así, que el $92.6 \%$ de la muestra declare que es necesario controlar el peso aunque el $73.7 \%$ no utilice estrategias para ello, hemos comprobado que corrobora los resultados de otros estudios (Amigo et al., 2005; Ballester y Guirado, 2003; Espina et al., 2001; Gómez-Peresmitré y Acosta, 2002; Sepúlveda et al., 2001). Respecto a la presencia en las mujeres de una mayor insatisfacción con el propio cuerpo, también nuestro estudio ha corroborado investigaciones anteriores (Bane y McAuley, 1998; Cash y Henry, 1995; Gómez-Peresmitré y Acosta, 2002; Heunemamm et al., 1966; Lameiras et al., 2003; Loland, 1998). Igualmente, el que los más jóvenes sean los que se encuentran más insatisfechos con su imagen, (Rodríguez-Fernández, 2008). Así como que los sujetos más satisfechos presenten, significativamente, una mayor autoestima, significa que una pobre imagen corporal se asocie con una baja autoestima, y coincide con los resultados del estudio de Calado et al. (2004).

Por todo esto, creemos que hay que seguir investigando los factores psicológicos y/o sociales que están incidiendo en generar o mantener conductas de riesgo en nuestra alimentación y en el control del peso. Desde nuestro punto de vista, factores psicológicos, como la autoestima, y sociales, como la imagen corporal, están 
directamente relacionados con los hábitos y estrategias que utilizamos a la hora de alimentarnos. Por ello, defendemos la necesidad de desarrollar programas de prevención e intervención psicoeducativos para abordar un fenómeno tan complejo y determinado por tantos factores.

\section{REFERENCIAS}

Acosta, M.V. y Gómez-Peresmitré, G. (2003). Insatisfacción corporal y seguimiento de dieta. Una comparación transcultural entre adolescentes de España y México. Internacional Journal of Clinical and Health Psychology, 3(1), 9-21.

Amigo, I., Fernández, C., Rodríguez, E. y Rodríguez, A. (2005). Creencias sobre las estrategias para el control del peso. Psicothema, 17(3), 418-421.

Ballester, R. y Guirado, M.C. (2003). Detección de conductas alimentarias de riesgo en niños de 11 a 14 años. Psicothema, 15(4), 556-562.

Bane, S. y McAuley, E. (1998). Body image and exorcice. En J. L. Duda (Ed.), Advances in Sport and Exercice Psychology Measurement (pp. 311-322). Morgantown, WV.

Baile, J.I. y Garrido, E. (1999). Autoimagen referente al peso en un grupo de chicas adolescentes. Anales del Sistema Sanitario Navarro, 22, 167-175.

Behar, M. e Icaza S. (1972). Nutrición. México: Interamericana.

Burgess, A. y Dean, R.F. (1963). La malnutrición y los hábitos alimentarios. USA: OPS/OMS.

Calado, M., Lameiras, M. y Rodríguez, Y. (2004). Influencia de la imagen corporal y la autoestima en la experiencia sexual de estudiantes universitarias sin trastornos alimentarios. International Journal of Clinical and Health Psychology, 4(2), 357-370.

Cash, T.F. (1994). Body-image attitudes: Evaluation, investment and affect. Perceptual and Motor Skills, 78(2), 1-1170.

Cash, T.F. y Pruzinsky, T.E. (1990). Body images: Development, deviance and change. New York: Guilford Press.

Cash, T.F. y Henry, P.E. (1995). Women's body images. The results of a national survey in the U.S.A. Sex rolex, 33, 19-28.

Cannon, W.B., y Washburn, A.L. (1912). An explanation of hunger. American Journal of Physiology, 29, 441- 454.

Espina, A., Ortego, M.A., Ochoa, I., Yenes, F. y Alemán, A. (2001). La imagen corporal en los trastornos alimentarios, Psicothema, 13(4), 533-538.

Fabian, L.J. y Thompson, J.K. (1989). Body image and eating disturbance in young females. International Journal of Eating Disorders, 8, 63-74.

Flores, M., Bresanni, R. y Elias, L. (1973). Factors and tactics influencing consumer food habits and patterns. En F. Byrnes, Potentials of field beans and food legumes in Latin América. Guatemala (pp. 88-102). Guatemala: INCAP.

Fox, K.R. (2000). The efects of exercice on self-perceptions and self-esteem. En S.J.H. Biddle, K.R. Fox y S.H. Boutcher (Eds.), Physical Activity and Psychological Well-Being (pp. 88-118). Londres: Routhledge.

Gázquez, J.J., Pérez-Fuentes, M.C., Fernández, M.M., González, L., Ruiz, M.I. y Díaz, A.M. (2009). Old-age stereotypes related to the gerontology education: an intergenerational study. European Journal of Education and Psychology, 2(3), 263-273.

Gómez-Peresmitré, G. y Acosta, M.V. (2002). Valoración de la delgadez. Un estudio transcultural (Mexico/España). Psicothema, 14(2), 221-226. 
Heunnmann, R.L., Shapiro, L.R., Hampton, M.C. y Mitchell, B.W. (1966). A longitudinal study of gross body composition and body conformation and their association with food and activity in teenage population. American Journal of Clinic Nutrion, 18, 325-338.

Hurtado, E. (1990). Aspectos socio-culturales relacionados con el consumo de alimentos en INCAP. Alimentación y nutrición en Centroamérica y Panamá: Análisis y estrategias para su desarrollo. Guatemala: INCAP.

Lameiras, M., Calado, M., Rodríguez, Y. y Fernández, M. (2003). Hábitos alimentarios e imagen corporal en estudiantes universitarios sin trastornos alimentarios. Revista Internacional de Psicología Clínica y de la Salud, 3(1), 23-33.

Leon, G.R., Fulkerson, J.A., Perry, C.L. y Cudeck, R. (1993). Personality and behavioural ulnerabilities associated with risk state for eating disorders in adolescent girls. Journal of Abnormal Psychology, 102, 138-444.

Loland, N.W. (1998). Body image and physical activity. A survey among Norwegian men and women. International Journal of Sport and Exercice Psychology, 29, 339-365.

López-Espinoza, A. (2007). Análisis experimental en conducta alimentaria. Anales de Psicología, 23(2), 258-263.

Martín-Albo, J., Núñez, J.L., Navarro, J.G. y Grijalvo, F. (2007). The Rosenberg Self-Esteem Scale: Translation and validation in university students. The Spanish Journal of Psychology, 10, 458-467.

Mellor, J.M., Rapoport, R.B. y Maliniak, D. (2008). The impact of child obesity on active parental consent in school-based survey research on healthy eating and physical activity. Evaluation Review, 32(3), 298-312.

Merino, H., Pombo, M.G. y Godás, A. (2001). Evaluación de las actitudes alimentarias y la satisfacción corporal en una muestra de adolescentes. Psicothema, 13(4), 539-545.

Moreno, J.A., Cervelló, E. y Moreno, R. (2008). Importancia de la práctica físico-deportiva y del género en el autoconcepto físico de los 9 a los 23 años. International Journal of Clinical and Health Psychology, 8(1), 171-183.

Mock, V. (1993). Body image in women treated with breast cancer. Nursing Research, 42, 153-157.

Pruzinsky, T. y Cash, T.F. (1990). Integrative themes in body-image development, deviance, and change. In T.F. Cash y T. Pruzinsky (Eds.), Body Images: Development, Deviance, and Change (pp. 337-349). N.Y.: Guilford Press.

Rodríguez, S. y Cruz, S. (2008). Insatisfacción corporal en adolescentes latinoamericanas y españolas. Psicothema, 20(1), 131-137.

Rodríguez-Fernández, A. (2008). El autoconcepto físico y el bienestar/malestar psicológico en la adolescencia. Revista de Psicodidáctica, 13(2), 155-158.

Rosenberg, M. (1965). Society and the adolescent self-image. Princeton, N.J.: Princeton University Press.

Salvador et al., (2008). "Cuestionario de Creencias y Estrategias para el Control del Peso". Manuscrito sin publicar. Grupo PESSO. Departamento de Psicología Evolutiva y de la Educación: Universidad de Almería.

Salvador, M., De la Fuente, M. y Alvarez, J.F. (2009). Las habilidades sociales en directores de centros escolares. European Journal of Education and Psychology, 2(3), 275-288.

Shavelson, R.J. y Bolus, R. (1992). Self-Concept: The interplay of theory and methods. Journal of Educational Psychology, 74, 3-17.

Sepúlveda, A.R., Botella, J. y León, J.A. (2001). La alteración de la imagen corporal en los trastornos de alimentación. Psicothema, 13(1), 7-16.

Turró, R. (1912). Orígenes del Conocimiento El Hambre. Barcelona: Minerva. 
Williamson, D.A., Womble, L.G, Zucker. N.L., Reas, D.L, White, M.A. y Blouin, D.C. y Greenway, F. (2000). Body image assessment for obesity (BIA-O): development of a new procedure. International Journal of Obesity Relatedt Metabolic Disorders, 24(10), 1326-1332.

Recibido: 2 de febrero de 2010

Recepción Modificaciones: 19 de agosto de 2010

Aceptado: 27 de agosto de 2010 